\title{
Possible role of biochemiluminescent photons for lysergic acid diethylamide (LSD)-induced phosphenes and visual hallucinations
}

Kapócs, Gábor ; Scholkmann, Felix ; Salari, Vahid ; Császár, Noémi ; Szőke, Henrik ; Bókkon, István

\begin{abstract}
Today, there is an increased interest in research on lysergic acid diethylamide (LSD) because it may offer new opportunities in psychotherapy under controlled settings. The more we know about how a drug works in the brain, the more opportunities there will be to exploit it in medicine. Here, based on our previously published papers and investigations, we suggest that LSD-induced visual hallucinations/phosphenes may be due to the transient enhancement of bioluminescent photons in the early retinotopic visual system in blind as well as healthy people.
\end{abstract}

DOI: https://doi.org/10.1515/revneuro-2016-0047

Posted at the Zurich Open Repository and Archive, University of Zurich

ZORA URL: https://doi.org/10.5167/uzh-207535

Journal Article

Published Version

Originally published at:

Kapócs, Gábor; Scholkmann, Felix; Salari, Vahid; Császár, Noémi; Szőke, Henrik; Bókkon, István (2017). Possible role of biochemiluminescent photons for lysergic acid diethylamide (LSD)-induced phosphenes and visual hallucinations. Reviews in the Neurosciences, 28(1):77-86.

DOI: https://doi.org/10.1515/revneuro-2016-0047 


\section{Possible role of biochemiluminescent photons for lysergic acid diethylamide (LSD)-induced phosphenes and visual hallucinations}

DOI 10.1515/revneuro-2016-0047

Received August 3, 2016; accepted September 3, 2016; previously published online October 12, 2016

\begin{abstract}
Today, there is an increased interest in research on lysergic acid diethylamide (LSD) because it may offer new opportunities in psychotherapy under controlled settings. The more we know about how a drug works in the brain, the more opportunities there will be to exploit it in medicine. Here, based on our previously published papers and investigations, we suggest that LSD-induced visual hallucinations/phosphenes may be due to the transient enhancement of bioluminescent photons in the early retinotopic visual system in blind as well as healthy people.
\end{abstract}

Keywords: LSD; ultra-weak photon emission; visual hallucinations/phosphenes; visual system.

\section{Introduction}

Lysergic acid is a natural substance from the parasitic rye fungus Claviceps purpurea. Lysergic acid diethylamide

*Corresponding author: István Bókkon, Psychoszomatic OutPatient Department, H-1037, Budapest, Hungary; and Vision Research Institute, Neuroscience and Consciousness Research Department, Lowell, MA 01854, USA, e-mail: bokkoni@yahoo.com

Gábor Kapócs: Social Home for Psychiatric Patients, H-9970, Szentgotthard, Hungary; and Institute of Behavioral Sciences, Semmelweis University, H-1089, Budapest, Hungary Felix Scholkmann: Biomedical Optics Research Laboratory, Department of Neonatology, University Hospital Zurich, University of Zurich, CH-8091 Zurich, Switzerland; and Research Office for Complex Physical and Biological Systems (ROCoS), CH-8038 Zurich, Switzerland

Vahid Salari: Department of Physics, Isfahan University of Technology, Isfahan 84156-83111, Iran; and School of Physics, Institute for Research in Fundamental Sciences (IPM), Tehran 19395-5531, Iran Noémi Császár: Psychoszomatic OutPatient Department, H-1037, Budapest, Hungary; and Gaspar Karoly University Psychological Institute, H-1091 Budapest, Hungary

Henrik Szőke: Doctors School of Health Sciences, University of Pécs, H-7621 Pécs, Hungary
(LSD) is a semisynthetic hallucinogenic drug of lysergic acid that was first synthesized by Albert Hofmann in 1938 (Hofmann, 1980). In the United States and western Europe, numerous experiments were performed from the 1950s up to the early 1970s by therapists and researchers that administered LSD to several thousands of people as a therapy for alcoholism, anxiety, depression, and cancer. Despite the numerous positive effects of LSD and its low toxicity, from the early 1970s onward, psychedelics were banned across the United States and worldwide (Das et al., 2016).

In the past two decades, the therapeutic use of LSD again increased concerning the therapeutic potential of LSD. A recent large population investigation of 130000 adults (including 19000 people that used psychedelic drugs) in the United States concluded that there is no link between the use of psychedelic LSD, psilocybin, or mescaline and mental health problems (Johansen and Krebs, 2015). Psychedelic nonaddictive LSD could prove beneficial to millions of people. However, more research has to be performed so that this promising drug can be successfully used for psychotherapy in the future (Das et al., 2016).

It is known that LSD causes visual hallucinations involving phosphenes (i.e. brief sensations of light). The mechanism of how LSD is causing this visual effect is, however, not completely understood. To this end, we present in this paper a unified explanation based on our previous published work that came to the conclusion that phosphenes may be due to the transient enhancement of bioluminescent photons in the visual system.

\section{Phosphenes}

The phosphene phenomenon is characterized by the sensation of light without photons actually entering the eye. Phosphene light perceptions (i.e. visions of geometric patterns and more complex phenomena such as spots or bars as well as disordered structures of colorless or colored lights) can be induced by means of mechanical, magnetic, 
or electrical stimulation of the visual system (Reznikov, 1981; Lindenblatt and Silny, 2002; Merabet et al., 2003; Tehovnik et al., 2009; Sczesny-Kaiser et al., 2016). Diverse drugs, stress, high-energy ionizing radiation, and highenergy particles and also optic nerve diseases can also elicit phosphenes. The phosphene sensation can emerge in a variety of diseases of the visual pathways, but healthy people can perceive them as well (Brigatti and Maguluri, 2005; Ashtari et al., 2014). Phosphene lights are only perceived by blind people that have prior visual experiences, suggesting that an early visual experience is essential to maintain any level of residual visual function (Merabet et al., 2003). The perceived phosphene lies within the visual hemifield contralateral to the stimulated cortical hemisphere and reflects the retinotopic structure of the visual cortex.

\section{Ultra-weak photon emission}

Numerous experiments have provided evidence that living cells continuously emit ultra-weak light without any excitation during natural metabolic processes (Scott et al., 1991; Cohen and Popp, 1997; Takeda et al., 1998; Kobayashi et al., 1999a, 2014, 2016; Kataoka et al., 2001; Tang and Dai, 2014a; Kobayashi, 2014; Alvermann et al., 2015; Kamal and Komatsu 2016). This ultra-weak photon emission (UPE) is also termed 'ultra-weak bioluminescence', 'low-intensity chemiluminescence', 'ultra-weak photons', 'biophotons', and others. UPE is mainly produced by several chemical reactions, mostly through bioluminescent radical reactions of reactive oxygen species (ROS) and reactive nitrogen species (RNS) as well as the deactivation of energetically excited molecules. These reactions are, for example, lipid peroxidations, peroxisomal reactions, reactions associated with the activity of the mitochondrial respiration chain, oxidation of catecholamines, oxidation of tyrosine and tryptophan residues in proteins, and others (Kruk et al., 1989; Nakano, 1989; Watts et al., 1995; Steele, 2003; Kamal and Komatsu, 2015). The UPE spectrum is in the range of about 200 to $800 \mathrm{~nm}$. It should be considered that the term 'ultra-weak photon emission' satisfies not completely the biological significance because the in vivo photon intensity can be basically higher inside cells compared to the measured photon emission ex vivo (Cliento, 1988; Slawinski, 1988; Bókkon et al., 2010). The measured UPE from macroscopic biological objects stems mainly from naturally occurring oxidation processes on the surface of the biological object (i.e. skin and cellular membranes; Blake et al., 2011). In addition, because isolated parts and biomolecules of cells can produce UPE (Vladimirov et al., 1966; Nakano, 1989; Devaraj et al., 1991; Scott et al., 1991), it also supports that the real intensity of UPE can be basically higher inside cells. That the in vivo photon emission rates (inside cells) are higher than the measured one is a prerequisite of our hypothesis presented in this manuscript. It is a prerequisite that is not unreasonable according to our view and understanding.

During normal metabolism, neurons show a continuous production of UPE through free radical reactions (Imaizumi et al., 1984; Isojima et al., 1995; Kataoka et al., 2001; Wang et al., 2011; Salari et al., 2015, 2016). It has been shown that UPE correlated with cerebral energy metabolism, cerebral blood flow (CBF), oxidative processes, and electrical activity in the rat brain in vivo (Kobayashi et al., 1999b), which implies that there can be neural activity-dependent UPE in the brain (Isojima et al., 1995). The latest experiments (Tang and Dai, 2014b) revealed that the glutamate-induced biophoton intensity reflects biophoton transmission along the axons and in neural circuits. It was demonstrated that photons associated with endogenous UPE could be conducted along neural fibers (Sun et al., 2010). A recently published computer simulation is supporting this conclusion (Kumar et al., 2016).

Generated biophotons can be absorbed by natural chromophores of cells and neurons (such as porphyrin and pyridinic rings, flavinic, and lipid chromophores; Kato et al., 1981; Karu, 1999; Mazhul' and Shcherbin, 1999) that generate electronically excited states, which may excite nearby molecules and trigger or regulate cellular signal processes.

\section{Phosphenes: possibly elicited by UPE}

Recently, a new biopsychophysical idea (Bókkon, 2008) was suggested about the phosphene phenomenon. It was proposed that retinal and cortical phosphenes can have similar mechanisms (i.e. both seem to be due to the endogenous free-radical reactions causing bioluminescent biophotons). Induced or spontaneous overproduction of free radicals and energetically excited molecules seem to create a brief increase of the generation of bioluminescent biophotons within neurons of the retinotopic visual system. It is hypothesized that, when this excess biophoton emission exceeds a distinct threshold, it then can become a conscious light sensation. The threshold value is unknown until now. Indirect evidence indicates 
that the threshold could be relatively low. Hecht et al. (1942) showed, for example, that dark-adapted human subjects are able to report light signals as low as a few photons ( $\sim 5-7)$. Tinsley et al. (2016) reported recently that a single-photon incident on the eye could be perceived by subjects. This suggests that intrinsic biophotons produced within the retinal system may produce retinal phosphene light perception (Császár et al. 2016). In the case of retinal phosphenes, we have to consider that the phototransduction enzyme cascade provides enormous signal amplification (Arshavsky and Burns, 2014). However, in the case of retinotopic visual areas, there is a different mechanism regarding phosphene generation that would require UPE among synchronized neurons. According to Tehovnik and Slocum (2007), the 'functional unit for phosphene induction in V1 is most likely the hypercolumn, which is about $1 \times 0.7 \mathrm{~mm}$ of tissue composed of layers spanning some $2 \mathrm{~mm}$ of tissue from the surface of cortex'. If we consider the calculations of Tehovnik and Slocum (2007) and the fact that the human cortex contains about 50000 neurons $/ \mathrm{mm}^{3}$ (Braitenberg and Schüz, 1998), it should be expected that a few hundred visual neurons to some thousand visual neurons are involved in the conscious phosphene perception in humans.

Concerning this prediction by Bókkon (2008), one specific type of phosphene phenomena (i.e. retinal phosphenes during space travel; Fuglesang et al., 2006) was found to follow this prediction according to experimental work of Narici et al. (2009), 2012, 2013). It has been shown that ionizing radiation (cosmic rays) induced free radicals that elicited reactions producing photon emission through retinal lipid peroxidation (Narici et al., 2009). These chemiluminescent photons are then absorbed by the photoreceptors and trigger the phototransduction cascade, causing the perception of phosphenes, similar to that induced by external light during normal vision.

Recently, we also pointed out (Császár et al., 2016) that cortical phosphenes seem to be due to the

1 This concept was based on the experiments of Tang and Dai (2014a,b) that provided evidence that the glutamate-induced UPE intensity reflected biophoton transmission along the axons and in neural network. Previously, it was proposed (Bókkon, 2008) that retinal and cortical phosphenes may have similar mechanisms: both seem to be related to UPE elicited by radical reactions. Regarding retinal phosphenes, they essential originate from excess free radicals causing lipid peroxidation (Narici et al., 2012, 2013) in the photoreceptors that can generate an excess UPE in the visual range. Regarding the cortical phosphenes, they may be originated from glutamate-induced redox processes. For example, tDCS induces electric currents that can produce membrane depolarization (during anodal stimulation, increased firing and excitability of the cortical neurons), increas- glutamate-related excess biophoton emission in the occipital cortex ${ }^{1}$. Our notion was based on the experiments of Tang and Dai (2014b) that provided evidence that the glutamate-induced biophoton intensity reflected biophoton transmission along the axons and in neural circuits.

We should also mention that we first suggested (Bókkon and Vimal, 2009) that not only phosphenes but also the discrete dark noise of retinal rods could be due to bioluminescent photons of lipid peroxidation. This notion was later fundamentally supported by experiments (Wang et al. 2011; Li and Dai, 2016) and calculations (Salari et al., 2016).

\section{Metabolism and distribution of LSD}

After oral administration, LSD is well absorbed from the gastrointestinal tract and is further distributed to different body tissues (Passie et al., 2008). The largest concentration of LSD has been found in the liver, where it is metabolized. According to in vitro studies, LSD is metabolized by NADH-dependent microsomal liver enzymes to inactive 2-oxy-LSD and 2-oxo-3-hydroxy LSD in humans. LSD can easily pass the blood-brain barrier (Axelrod et al. 1957), so that it can easily enter the brain. Snyder and Reivich (1966) investigated the regional distribution of LSD in squirrel monkey brains and revealed that the pituitary and pineal glands presented the highest LSD concentrations compared to the cortex. The limbic system (amygdale, hippocampus, fornix, and septal region) had about three times more LSD absorbed than the cortical structures. Regarding cortical areas, LSD mainly concentrated in the visual and auditory regions, hypothalamus, extrapyramidal system, and thalamus. The brain stem

ing glutamate release that activates the NMDA receptors. Activated NMDA receptors enhance the NADPH oxidase activity and subsequently an increased mitochondrial free-radical production. This transitory free-radical production can generate additional UPE in the visual cortex that finally generates the phosphene perception in the subject - so our hypothesis. Furthermore, TMS can transiently disrupt and modulate neural activity in local brain areas possibly also involving UPE. TMS inhibition possibly reflects the activity of GABAergic interneurons, but facilitation depends on the activation of intracortical fibers by the subthreshold stimulus, inducing the local release of glutamate (Oliveri and Caltagirone, 2006). The TMS-induced electric field could induce phosphenes in the striate cortex (V1) of the macaque (Tehovnik and Slocum, 2007) and selective stimulated V1 and V2 (Salminen-Vaparanta et al., 2014) that were able to elicit phosphene perception to a similar degree. It seems that TMS-induced phosphenes are also due to glutamate-related UPE. 
had similar LSD concentrations like the cortex. It was also found that LSD was equally distributed between the gray and white matter.

\section{LSD pharmacology: a short review}

For an excellent review about the pharmacology of LSD, see Nichols (2004). To support our arguments presented in this paper, a few specific facts of LSD metabolism should be mentioned. LSD can bind to various monoamine receptors, such as serotonin $(5-\mathrm{HT})_{1 \mathrm{~A} / \mathrm{B} / 1 \mathrm{D}}, 5-\mathrm{HT}_{2 \mathrm{~A} / 2 \mathrm{C}}$, $5-\mathrm{HT}_{5 \mathrm{~A}}, 5-\mathrm{HT}_{6}$, and $5-\mathrm{HT}_{7}, \mathrm{D}_{1}$ and $\mathrm{D}_{2}$ dopamine receptors, and $\alpha_{1}-$ and $\alpha_{2}$-adrenergic receptors (Nichols, 2004). 5-HT is produced in the raphe nuclei of the brain stem. Because LSD has a similar molecular structure as 5 -HT, it can specially act on $5-\mathrm{HT}_{2}$ receptors. Glutamate is the major excitatory neurotransmitter in the nervous system. It is demonstrated that LSD enhances glutamatergic transmission. When LSD stimulates $5-\mathrm{HT}_{2 \mathrm{~A}}$ receptors on glutamate axon projections from the thalamus, it excites the cortical pyramidal cells that release glutamate (Nichols, 2004). Dopamine is produced in numerous regions of the brain, including the substantia nigra and the ventral tegmental area. LSD can also directly activate dopamine pathways. It has been revealed that 5 -HT regulates the major inhibitory neurotransmitter $\gamma$-aminobutyric acid (GABA) interneurons (Abi-Saab et al., 1999; Liu, et al., 2000). In addition, LSD can activate the noradrenaline [norepinephrine (NE)] neurons located in the locus coeruleus of the brain. The discovery of D2L-5- $\mathrm{HT}_{2 \mathrm{~A}}$ and $5-\mathrm{HT}_{2 \mathrm{~A}} \mathrm{R}$-metabotropic glutamate receptor (mGluR) 2 heteroreceptor complexes further complicates the mechanism of action of LSD in the brain. mGluR2/3 ligands can modulate the responses induced by the activation of $5-\mathrm{HT}_{2 \mathrm{~A}} \mathrm{Rs}$ (Moreno et al. 2011). D2L-5-HT $\mathrm{HA}_{2 \mathrm{~A}}$ also shows receptor-receptor interactions (Borroto-Escuela et al., 2014).

We agree with Nichols' (2004) notion that 'hallucinogens enhance sensitivity/excitability of the cortical processing while at the same time causing glutamate to be released from thalamic afferents that normally signal incoming sensory information to be processed'. However, according to experiments, $5-\mathrm{HT}_{2 \mathrm{~A}}$ heteroreceptors on thalamocortical neurons induce an enhanced release of glutamate from cortical neurons a common mechanism in the action of hallucinogens (Kharazia and Weinberg, 1994; Scruggs et al., 2003; Muschamp et al., 2004).

\section{Effects of LSD in the visual cortex are identical to the effects of external light: LSD-induced visual experiences in blind people}

Although retinal as well as cortical processes can contribute to LSD-induced visual hallucinations (Brust, 2004), they can also occur without a functioning retina. Krill et al. (1963) reported on LSD-induced visual experiences in blind people. Thirteen of 24 subjects (some with bilateral enucleations) reported LSD-induced visual hallucinations. Krill et al. concluded that it would be 'evident that a normal retina is not needed for the occurrence of LSD-induced visual experiences. These visual experiences do not seem to differ from the hallucinations reported by normal subjects after LSD.' In addition, these LSD-induced visual hallucinations emerged only in blind people who had some kind of prior visual activities. Some blind people did not report LSD-induced visual hallucinations because they never had sufficient visual experience to develop a proper functioning visual system [by the way, based on monocular deprivation experiments, Hubel and Wiesel (1965) proved that proper visual system development requires visual experience]. Alema (1952) also reported that orally administered $50 \mu \mathrm{g}$ LSD produced complex visual hallucinations in a subject with bilateral enucleations of the eyeball.

Lately, Carhart-Harris et al. (2016) were the first who visualized the effects of LSD on the human brain using functional magnetic resonance imaging (fMRI) and magnetoencephalography (MEG) in healthy volunteers. All volunteers with closed eyes reported about visual hallucinations and significant changes in consciousness under the influence of LSD. The major findings were an increased CBF, decreased $\alpha$ power in the visual cortex, and an expanded primary visual cortex (V1) functional connectivity [expanded V1 resting state functional connectivity (RSFC)], which were correlated with the ratings of visual hallucinations. In other words, blood streamed to the visual cortex and neural connections within the visual cortex are strengthened, and the stronger they were, the more visual hallucinators were experienced by volunteers. However, these properties mentioned in general correlate during external visual stimulation. Carhart-Harris et al. (2016) revealed that the effects of LSD in the visual cortex were identical to the effects of external light (photons) striking the retina. They also found that LSD produced (compared to placebo) an 
unusual event as disparate areas within the brain communicate with each other what they normally not do. The visual cortex particularly increased its communication with other brain areas, but there was a decreased connectivity between the parahippocampus and retrosplenial cortex (RSC).

Roseman et al. (2016) investigated the RSFC of 10 healthy voluntaries under the influence of LSD relative to placebo. Researchers assumed that eyes-closed psychedelic hallucinations (visions of geometric patterns and more complex phenomena) might produce transient local retinotopic activation, which are naturally associated with visual stimulation. The results indicated that, under LSD, with eyes closed, the early visual system (V1 and V3) works as if it were seeing spatially localized external visual inputs.

\section{LSD oxidation triggers chemiluminescent reactions via ROS}

LSD is mostly metabolized to 2-oxo-3-hydroxy-LSD (O-HLSD) and $N$-desmethyl-LSD (nor-LSD) by means of cytochrome $P 450$ complex liver enzymes in vivo. Recently, Gomes et al. (2012) revealed that LSD could also be a substrate for horseradish peroxidase (HRP) and myeloperoxidase (MPO). They found that HRP and MPO peroxidases could metabolize LSD to the major metabolites observed in vivo. In addition, the reactions of LSD with the peroxidases were chemiluminescent and sensitive to inhibition by reactive oxygen scavengers, which indicated that the classic peroxidase cycle is involved in this new alternative metabolic pathway.

Furthermore, LSD metabolism by the HRP/hydrogen peroxide $\left(\mathrm{H}_{2} \mathrm{O}_{2}\right)$ system presented high chemiluminescence. LSD oxidation by phorbol 12-myristate 13-acetate (PMA)-activated neutrophils produced smaller chemiluminescence that was dependent on the addition of all the reagents (the intensity of the photon emission was about $10 \%$ of the emission compared to the $\mathrm{LSD} / \mathrm{HRP} / \mathrm{H}_{2} \mathrm{O}_{2}$ system).

However, MPO is not only expressed in immune cells as neutrophils and monocytes (Malle et al., 2007) but also by neurons (Green et al., 2004) and microglia (monocytederived cells), which represent roughly $5-10 \%$ of the cells found in normal brain (Lefkowitz and Lefkowitz, 2008). Green et al. (2004) revealed that numerous neuronal cell lines as well as primary neuronal cultures expressed MPO protein. Although the expression of MPO is increased in Alzheimer's disease brains, there was also a low-level expression of MPO in hippocampal pyramidal neurons in control tissues and cultured neuronal cells, which support that MPO can perform an essential physiological function in the brain.

\section{Summary}

Based on the discussed findings, the following conclusion/summary can be given:

- LSD can induce visual phosphenes and hallucinations that can occur without a functioning retina.

- LSD can induce visual phosphenes and hallucinations that emerge only in blind people who had some kind of prior visual experience (Krill et al., 1963).

- LSD-induced visual experiences do not seem to differ from the hallucinations reported by normal subjects after LSD.

- The effects of LSD in the visual cortex are identical to the effects of external light (photons) striking the retina (Krill et al., 1963; Carhart-Harris et al., 2016).

- LSD administration increases CBF to the visual cortex, and neural connections within the visual cortex are strengthened. The stronger they were, the more visual hallucinations were experienced by volunteers (Carhart-Harris et al., 2016).

- The eyes-closed psychedelic imagery (visions of geometric patterns; i.e. complex phosphenes and more complex visual scenes) may produce transient local retinotopic activations, which naturally are associated with visual stimulation. It was revealed that, under LSD, with eyes closed, the early visual system (V1 and V3) works as if it were seeing spatially localized external visual inputs (Roseman et al., 2016).

- The reactions of LSD with the peroxidases are chemiluminescent and sensitive to inhibition by reactive oxygen scavengers (Gomes et al., 2012).

- MPO is not only expressed in immune cells as neutrophils and monocytes (Malle et al., 2007) but also by neurons (Green et al., 2004) and microglia.

- The latest experiments (Tang and Dai, 2014b) support that the cortical phosphene lights are due to the glutamate-related excess biophoton emission in the occipital cortex (Császár et al., 2016).

- When LSD stimulates $5-\mathrm{HT}_{2 \mathrm{~A}}$ receptors on glutamate axon projections from the thalamus, it can excite the cortical pyramidal cells that release glutamate (Nichols, 2004). 
- $5-\mathrm{HT}_{2 \mathrm{~A}}$ heteroreceptors on thalamocortical neurons that induce the enhanced release of glutamate from cortical neurons is a common mechanism in the action of hallucinogens (Kharazia and Weinberg, 1994; Scruggs et al., 2003; Muschamp et al., 2004).

As we have previously pointed out (Császár et al., 2016), there are some studies recently published (Kobayashi et al., 1999a,b; Tang and Dai, 2014b) that may support that the conclusion that cortical phosphenes are due to the glutamate-related excess biophoton emission in the retinotopic early visual parts. Regarding cortical areas, LSD mainly concentrated in the visual regions. LSD by means of complex neurotransmitter and other processes can also create glutamate release from the excited cortical pyramidal cells and induce excess UPE in the early visual areas. In addition, LSD may also generate biochemiluminescent photons (i.e. drug-induced excess biophoton emission) in the visual regions of the brain via MPO. The special strong visual hallucinogenic effect (i.e. simple and complex phosphenes and visual hallucinations) of LSD may be due to the fact that there are several sources of UPE (biochemiluminescent)-producing mechanisms by LSD in the brain in parallel, especially in the early visual system (Figure 1).

One could argue, however, that the addition of LSD would enhance the production rate of biochemiluminescent photons to a negligible degree as, in the experimental work of Gomes et al. (2012), the chemiluminescent properties of LSD were detected when adding LSD in the millimolar range, whereas in cells itself there was already enough substrate available (in the molar range) for chemiluminescence reactions. However, it has to be taken into account that the experimental set-up of Gomes et al. prevented the detection of weak photon emission (i.e. a luminometer was used instead of a high-sensitive photomultiplier, for example); thus, the real photon production rate of LSD might be underestimated due to the experimental set-up. In addition, the application of a few micromolars of LSD may still be enough to trigger sufficient photon generation reactions in vivo that cause cell physiological processes.

There are various experiments related to transcranial magnetic stimulation (TMS)-induced phosphenes in early and late blind people. Cattaneo and Vecchi (2011) concluded that, 'however, to date, no cases have been reported of visual phosphenes induced in congenitally blind subjects' regarding TMS. LSD can induce visual hallucinations/phosphenes only in blind people who had some kind of prior visual activities. TMS can also induce visual hallucinations/phosphenes only in blind people who had prior visual experiences. It suggests that there may have some common mechanisms of action between LSD-induced visual hallucinations/phosphenes and the TMS-induced phosphenes (Bókkon, 2008; Császár et al., 2016). Namely, both methods can induce visual hallucinations/phosphenes only in blind people who had prior visual activities. TMS induces and transcranial direct current stimulation (tDCS) presents electric currents in the brain, which increase neuronal excitability in the stimulated area that can create the local release of glutamate that generates excess UPE in the early visual parts, and finally can produce phosphene light perception. In addition, both TMS and TDCS can also perform a nonspecific effect on the 5-HT system and 5- $\mathrm{HT}_{2 \mathrm{~A}}$ receptors (Gur et al., 2000; Baeken et al., 2011; Miniussi et al., 2013) similarly as LSD.

We should also mention the experiments by Knoll et al. (1963). They induced phosphenes by simultaneous electrical and chemical (by hallucinogenic mescaline, psilocybin, and LSD) stimulation. Hallucinogenic chemical stimulation could increase the intensity, production, and complexity of electrically induced phosphene patterns, which may support our notion that biochemiluminescent neurochemical processes may be a common base of chemical (by LSD) or current (by TMS or tDCS) induced visual hallucinations/phosphenes.

One could argue that the environmental light (sunlight or light from lamps) may cause phosphenes as well. The optical properties of the skin tissue, skull, and cerebrospinal fluid enable light from external stimuli to reach the cortical tissue especially in the 'optical window' (Scholkmann et al., 2014). In principle, light irradiated into the head can traverse the skull and have the possibility to modulate biochemical and biophysical functions within the brain. In our suggested hypothesis, we emphasize that our proposed visual cortical phosphene perception mechanism is due to the UPE inside neurons. Our opinion is that externally generated photons that traverse the skull hardly can produce phosphene perception. The reason for this might be found in the fact that the endogenously produced light may have specific features (spectrum, modulation, etc.) that differ from the light from the environment (Laager, 2015), preventing the triggering of phosphenes under normal environmental light conditions.

In summary, LSD-induced visual hallucinations/ phosphenes may be due to the transient enhancement of bioluminescent photons in the early retinotopic visual system in blind as well as healthy people. The special strong visual hallucinogenic effect (simple and complex phosphenes and visual hallucinations) of LSD may be due to the fact that there are more simultaneous sources of UPE (biochemiluminescent)-producing mechanisms by 


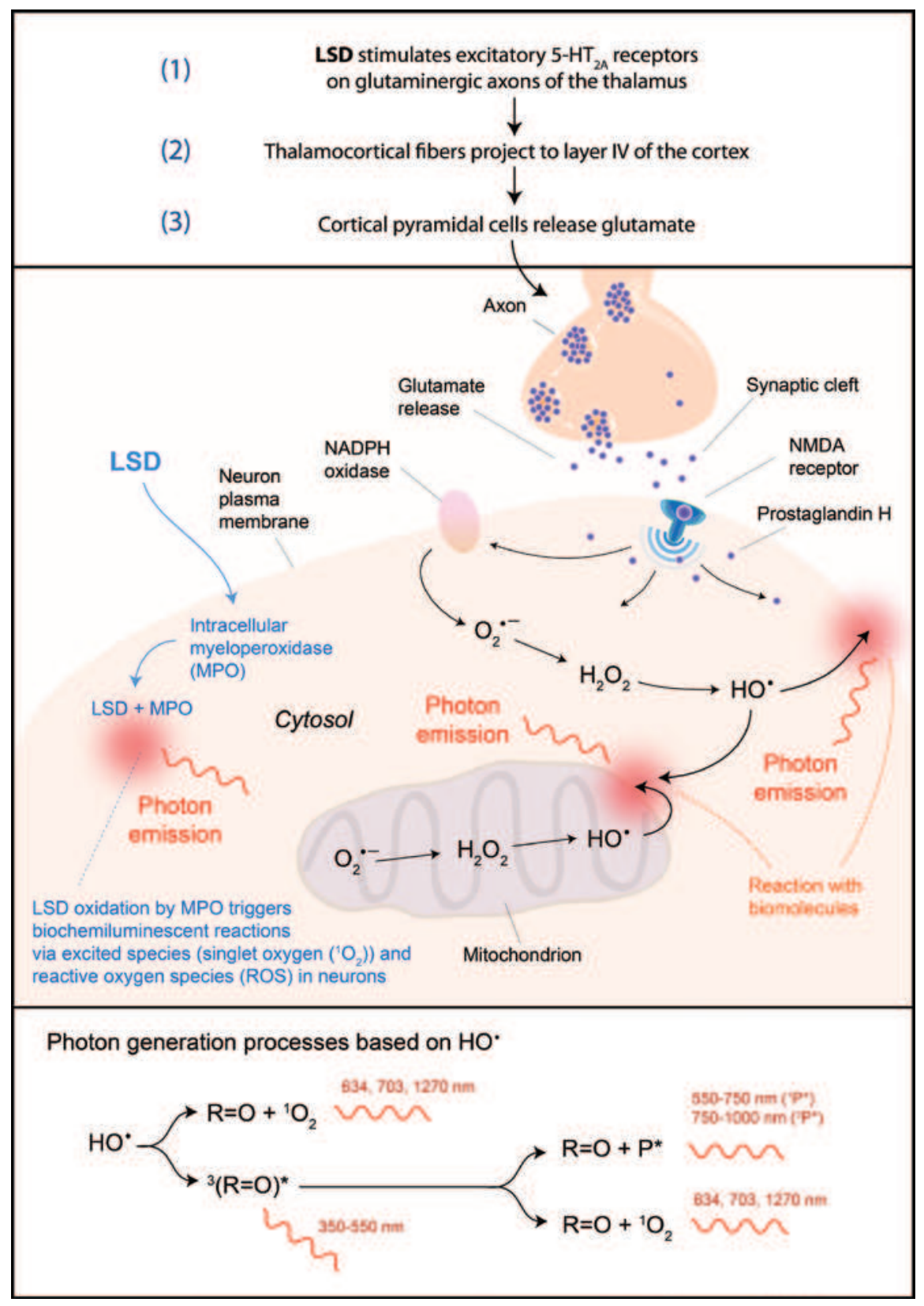

Figure 1: Visualization of the processes underlying the presented hypothesis.

The strong visual hallucinogenic effect (simple and complex phosphenes and visual hallucinations) of LSD may be due to different sources of UPE (biochemiluminescent) mechanisms induced by LSD in the brain, especially in the early visual system. The signal cascade is as follows: (1) LSD stimulates excitatory $5-\mathrm{HT}_{2 \mathrm{~A}}$ receptors on glutaminergic axons of the thalamus that induce the enhanced release of glutamate from cortical neurons. Next, the glutamate release activates NMDA receptors that stimulate NADPH oxidase activity and a free radical production. Finally, a temporary excess free-radical production can generate UPE in the visual cortex. (2) LSD is metabolized by MPO that produces biochemiluminescent photons (i.e. drug-induced excess UPE) in the visual regions of the brain. Superoxide anion radicals $\left(\mathrm{O}_{2} \cdot{ }^{-}\right)$are generated by the NADPH oxidase and mitochondrial metabolism. In subsequent reactions, $\mathrm{H}_{2} \mathrm{O}_{2}$ and finally hydroxyl radicals (HO.) are generated. $\mathrm{HO}$. reacts with biomolecules (lipids, proteins, and nucleic acids) that are part of the photon-generating processes. A summary of the main reactions is shown at the bottom of the figure. These reactions involve ground carbonyls $(R=0)$, singlet oxygen $\left({ }^{1} \mathrm{O}_{2}\right)$, singlet and triplet excited pigments $\left({ }^{1} \mathrm{P}^{\star},{ }^{3} \mathrm{P}^{\star}\right)$, and triple excited carbonyls $\left({ }^{3}(\mathrm{R}=0)^{\star}\right)$. For a detailed description of these reactions, see Pospisil et al. (2014).

means of LSD in the brain. In addition, LSD can exert effect through a specific neurochemical manner - compared to the nonspecific currents of TMS or tDCS - because LSD has a similar molecular structure as 5-HT. This specific effect may offer such opportunities, regarding induced hallucinations/phosphenes, in psychotherapy under controlled 
settings, which will probably not be achieved with devices such as the TMS or tDCS.

We emphasize that the UPE notion of phosphenes or complex hallucinations, retinal dark noise, and several visual phenomena (Bókkon, 2008; Bókkon and Vimal, 2009; Bókkon et al., 2011) should be really considered as a scientifically appropriate and provable mechanism to really understand the various visual phenomena in the future.

Conflict of interest statement: The authors declare no conflicts of interest. The authors alone are responsible for the content.

\section{References}

Abi-Saab, W.M., Bubser, M., Roth, R.H., and Deutch, A.Y. (1999). $5-\mathrm{HT}_{2}$ receptor regulation of extracellular GABA levels in the prefrontal cortex. Neuropsychopharmacology 20, 92-96.

Alema, G. (1952). Allucinazioni da acido lisergico in cieco sen a bulbi ocular. Riv. Neurol. 22, 720-733.

Alvermann, M., Srivastava, Y.N., Swain, J., and Widom, A. (2015). Biological electric fields and rate equations for biophotons. Eur. Biophys. J. 44, 165-170.

Arshavsky, V.Y. and Burns, M.E. (2014). Current understanding of signal amplification in phototransduction. Cell. Logistics 4, e28680.

Ashtari, M., Cyckowski, L., Yazdi, A., Marshal, K., Viands, A., Bókkon, I., Maguire, A., and Bennett, J. (2014). fMRI of retinal originated phosphenes experienced by patients with Leber congenital amaurosis. PLoS ONE 9, e86068.

Axelrod, J., Brady, R.O., Witkop, B., and Evarts, E.V. (1957). The distribution and metabolism of LSD. Ann. N. Y. Acad. Sci. 66, 435-444.

Baeken, C., De Raedt, R., Bossuyt, A., Van Hove, C., Mertens, J., Dobbeleir, A., Blanckaert, P., and Goethals, I. (2011). The impact of HF-rTMS treatment on serotonin (2A) receptors in unipolar melancholic depression. Brain Stimul. 4, 104-111.

Blake, T., Dotta, B.T., Buckner, C.A., Cameron, D., Lafrenie, R.M., and Persinger, M.A. (2011). Biophoton emissions from cell cultures: biochemical evidence for the plasma membrane as the primary source. Gen. Physiol. Biophys. 30, 301-309.

Bókkon, I. (2008). Phosphene phenomenon: a new concept. Biosystems 92, 168-174.

Bókkon, I. and Vimal, R.L.P. (2009). Retinal phosphenes and discrete dark noises in rods: a new biophysical framework. J. Photochem. Photobiol. B Biol. 96, 255-259.

Bókkon, I., Salari, V., Tuszynski, J., and Antal, I. (2010). Estimation of the number of biophotons involved in the visual perception of a single-object image: biophoton intensity can be considerably higher inside cells than outside. J. Photochem. Photobiol. B Biol. 100, 160-166.

Bókkon, I., Vimal, R.L.P., Wang, C., Dai, J., Salari, V., Grass, F., and Antal, I. (2011). Visible light induced ocular delayed bioluminescence as a possible origin of negative afterimage. J. Photochem. Photobiol. B Biol. 103, 192-199.
Borroto-Escuela, D.O., Romero-Fernandez, W., Narvaez, M., Oflijan, J., Agnati, L.F., and Fuxe, K. (2014). Hallucinogenic 5- $\mathrm{HT}_{2 \mathrm{~A}} \mathrm{R}$ agonists LSD and DOI enhance dopamine D2R protomer recognition and signaling of $\mathrm{D} 2-5-\mathrm{HT}_{2 \mathrm{~A}}$ heteroreceptor complexes. Biochem. Biophys. Res. Commun. 443, 278-284.

Braitenberg, V. and Schüz, A. (1998). Cortex: Statistics and Geometry of Neuronal Connectivity. 2nd ed. Berlin: Springer.

Brigatti, L. and Maguluri, S. (2005). Reproducibility of self-measured intraocular pressure with the phosphene tonometer in patients with ocular hypertension and early to advanced glaucoma. J. Glaucoma 14, 36-39.

Brust, J.C.M. (2004). Neurological Aspects of Substance Abuse. 2nd ed. USA: Elsevier, Inc. p. 260.

Carhart-Harris, R.L., Muthukumaraswamy, S., Roseman, L., Kaelen, M., Droog, W., Murphy, K., Tagliazucchi, E., Schenberg, E.E., Nest, T., Orban, C., et al. (2016). Neural correlates of the LSD experience revealed by multimodal neuroimaging. Proc. Natl. Acad. Sci. U. S. A. 113, 4853-4858.

Cattaneo, Z. and Vecchi, T. (2011). Blind Vision: The Neuroscience of Visual Impairment. (Cambridge, MA: MIT Press), p. 98.

Cliento, G. (1988). Photobiochemistry without light. Experientia 44 , 572-576.

Cohen, S. and Popp, F.A. (1997). Biophoton emission of the human body. J. Photochem. Photobiol. B Biol. 40, 187-189.

Császár, N., Scholkmann, F., Salari, V., Szőke, H., and Bókkon, I. (2016). Phosphene perception is due to the ultra-weak photon emission produced in various parts of the visual system: glutamate in the focus. Rev. Neurosci. 27, 291-299.

Das, S., Barnwal, P., Ramasamy, A., Sen, S., and Mondal, S. (2016). Lysergic acid diethylamide: a drug of 'use'? Ther. Adv. Psychopharmacol. 6, 214-228.

Devaraj, B., Scott, R.Q., Roschger, P., and Inaba, H. (1991). Ultraweak light emission from rat liver nuclei. Photochem. Photobiol. 54, 289-293.

Fuglesang, C., Narici, L., Picozza, P., and Sannita, W.G. (2006). Phosphenes in low earth orbit: survey responses from 59 astronauts. Aviat. Space Environ. Med. 77, 449-452.

Gomes, M.M., Dörr, F.A., Catalani, L.H., and Campa, A. (2012). Oxidation of lysergic acid diethylamide (LSD) by peroxidases: a new metabolic pathway. Forensic Toxicol. 30, 87-97.

Green, P.S., Mendez, A.J., Jacob, J.S., Crowley, J.R., Growdon, W., Hyman, B.T., and Heinecke, J.W. (2004). Neuronal expression of myeloperoxidase is increased in Alzheimer's disease. J. Neurochem. 90, 724-733.

Gur, E., Lerer, B., Dremencov, E., and Newman, M.E. (2000). Chronic repetitive transcranial magnetic stimulation induces subsensitivity of presynaptic serotonergic autoreceptor activity in rat brain. NeuroReport 11, 925-929.

Hecht, S., Schlaer, S., and Pirenne, M.H. (1942). Energy, quanta and vision. J. Opt. Soc. Am. 38, 196-208.

Hofmann, A. (1980). LSD: My Problem Child. New York, NY: McGrawHill.

Hubel, D.H. and Wiesel, T.N. (1965). Binocular interaction in striate cortex of kittens reared with artificial squint. J. Neurophysiol. 28, 1041-1059.

Imaizumi, S., Kayama, T., and Suzuki, J. (1984). Chemiluminescence in hypoxic brain - the first report. Correlation between energy metabolism and free radical reaction. Stroke 15, 1061-1065. 
Isojima, Y., Isoshima, T., Nagai, K., Kikuchi, K., and Nakagawa, H. (1995). Ultraweak biochemiluminescence detected from rat hippocampal slices. NeuroReport 6, 658-660.

Johansen, P.Ø. and Krebs, T.S. (2015). Psychedelics not linked to mental health problems or suicidal behavior: a population study. J. Psychopharmacol. 29, 270-279.

Kamal, A.H. and Komatsu, S. (2015). involvement of reactive oxygen species and mitochondrial proteins in biophoton emission in roots of soybean plants under flooding stress. J. Proteome Res. 14, 2219-2236.

Kamal, A.H. and Komatsu, S. (2016). Proteins involved in biophoton emission and flooding-stress responses in soybean under light and dark conditions. Mol. Biol. Rep. 43, 73-89.

Karu, T. (1999). Primary and secondary mechanisms of action of visible to near-IR radiation on cells. J. Photochem. Photobiol. B 49, 1-17.

Kataoka, Y., Cui, Y., Yamagata, A., Niigaki, M., Hirohata, T., Oishi, N., and Watanabe, Y. (2001). Activity-dependent neural tissue oxidation emits intrinsic ultraweak photons. Biochem. Biophys. Res. Commun. 285, 1007-1011.

Kato, M., Shinzawa, K., and Yoshikawa, S. (1981). Cytochrome oxidase is a possible photoreceptor in mitochondria. J. Photochem. Photobiol. 2, 263-269.

Kharazia, V.N. and Weinberg, R.J. (1994). Glutamate in thalamic fibers terminating in layer IV of primary sensory cortex. J. Neurosci. 14, 6021-6032.

Knoll, M., Kugler, J., Höfer, O., and Lawder, S.D. (1963). Effects of chemical stimulation of electrically-induced phosphenes on their bandwidth, shape, number and intensity. Confin. Neurol. 23, 201-226.

Kobayashi, M. (2014). Highly sensitive imaging for ultra-weak photon emission from living organisms. J. Photochem. Photobiol. B. 139, 34-38.

Kobayashi, M., Takeda, M., Ito, K., Kato, H., and Inaba, H. (1999a). Two-dimensional photon counting imaging and spatiotemporal characterization of ultraweak photon emission from a rat's brain in vivo. J. Neurosci. Methods 93, 163-168.

Kobayashi, M., Takeda, M., Sato, T., Yamazaki, Y., Kaneko, K., Ito, K., Kato H., and Inaba, H. (1999b). In vivo imaging of spontaneous ultraweak photon emission from a rat's brain correlated with cerebral energy metabolism and oxidative stress. Neurosci. Res. 34, 103-113.

Kobayashi, K., Okabe, H., Kawano, S., Hidaka, Y., and Hara, K. (2014). Biophoton emission induced by heat shock. PLoS ONE 9, e105700.

Kobayashi, M., Iwasa, T., and Tada, M. (2016). Polychromatic spectral pattern analysis of ultra-weak photon emissions from a human body. J. Photochem. Photobiol. B. 159, 186-190.

Krill, A.E., Alpert, H.J., and Ostfeld, A.M. (1963). Effects of a hallucinogenic agent in totally blind subjects. Arch. Ophthalmol. 69, 180-185.

Kruk, I., Lichszteld, K., Michalska, T., Wronska, J., and Bounias, M. (1989). The formation of singlet oxygen during oxidation of catechol amines as detected by infrared chemiluminescence and spectrophotometric method. Z. Naturforsch. C 44, 895-900.

Kumar, S., Boone, K., Tuszynski, J., Barclay, P.E., and Simon, C. (2016). Possible existence of optical communication channels in the brain. arXiv:1607.02969.

Laager, F. (2015). Light based cellular interactions: hypotheses and perspectives. Front. Phys. http://dx.doi.org/10.3389/ fphy.2015.00055.
Lefkowitz, D. and Lefkowitz, S. (2008). Microglia and myeloperoxidase: a deadly partnership in neurodegenerative disease. Free Radic. Biol. Med. 45, 726-731.

Li, Z. and Dai, J. (2016). Biophotons contribute to retinal dark noise. Neurosci. Bull. 32, 246-252.

Lindenblatt, G. and Silny, J. (2002). Electrical phosphenes: on the influence of conductivity inhomogeneities and small-scale structures of the orbita on the current density threshold of excitation. Med. Biol. Eng. Comput. 40, 354-359.

Liu, R., Jolas, T., and Aghajanian, G. (2000). Serotonin 5-HT(2) receptors activate local GABA inhibitory inputs to serotonergic neurons of the dorsal raphe nucleus. Brain Res. 73, 34-45.

Malle, E., Furtmuller, P., Sattler, W., and Obinger, C. (2007). Myeloperoxidase: a target for new drug development? Br. J. Pharmacol. 152, 838-854.

Mazhul', V.M. and Shcherbin, D.G. (1999). Phosphorescent analysis of lipid peroxidation products in liposomes. Biofizika 44, 676-681.

Merabet, L.B., Theoret, H., and Pascual-Leone, A. (2003). Transcranial magnetic stimulation as an investigative tool in the study of visual function. Am. J. Optom. Physiol. Opt. 80, 356-368.

Miniussi, C., Paulus, W., and Rossini P.M. (2013). Transcranial Brain Stimulation. (London: CRC Press, Taylor \& Francis Group).

Moreno, J.L., Holloway, T., Albizu, L., Sealfon, S.C., and GonzálezMaeso, J. (2011). Metabotropic glutamate $\mathrm{mGlu} 2$ receptor is necessary for the pharmacological and behavioral effects induced by hallucinogenic $5-\mathrm{HT}_{2 \mathrm{~A}}$ receptor agonists. Neurosci. Lett. 493, 76-79.

Muschamp, J.W., Regina, M.J., Hull, E.M., Winter, J.C., and Rabin, R.A. (2004). Lysergic acid diethylamide and [-]-2,5-dimethoxy4-methylamphetamine increase extracellular glutamate in rat prefrontal cortex. Brain Res. 1023, $134-140$.

Nakano, M. (1989). Low-level chemiluminescence during lipid peroxidations and enzymatic reactions. J. Biolumin. Chemilum. 4, 231-240.

Narici, L., De Martino, A., Brunetti, V., Rinaldi, A., Sannita, W.G., and Paci, M. (2009). Radicals excess in the retina: a model for light flashes in space. Radiat. Meas. 44, 203-205.

Narici, L., Paci, M., Brunetti, V., Rinaldi, A., Sannita, W.G., and De Martino, A. (2012). Bovine rod rhodopsin. 1. Bleaching by luminescence in vitro by recombination of radicals from polyunsaturated fatty acids. Free Radic. Biol. Med. 53, 482-487.

Narici, L., Paci, M., Brunetti, V., Rinaldi, A., Sannita, W.G., Carozzo, S., and Demartino, A. (2013). Bovine rod rhodopsin: 2. Bleaching in vitro upon ${ }^{12} \mathrm{C}$ ions irradiation as source of effects as light flash for patients and for humans in space. Int. J. Radiat. Biol. 89, 765-769.

Nichols, D.E. (2004). Hallucinogens. Pharmacol. Ther. 101, 131-181. Oliveri, M. and Caltagirone, C. (2006). Suppression of extinction with TMS in humans: from healthy controls to patients. Behav. Neurol. 17, 163-167.

Passie, T., Halpern, J.H., Stichtenoth, D.O., Emrich, H.M., and Hintzen, A. (2008). The pharmacology of lysergic acid diethylamide: a review. CNS Neurosci. Ther. 14, 295 -314.

Pospisil, P., Prassad, A., and Rac, M. (2014). Role of reactive oxygen species in ultra-weak photon emission in biological systems. J. Photochem. Photobiol. B Biol. 139, 11-23.

Reznikov, I.u.E. (1981). Mechanophosphene in optic nerve changes [in Russian]. Oftalmol. Z. 36, 218-220. 
Roseman, L., Sereno, M.I., Leech, R., Kaelen, M., Orban, C., McGonigle, J., Feilding, A., Nutt, D.J., and Carhart-Harris, R.L. (2016). LSD alters eyes-closed functional connectivity within the early visual cortex in a retinotopic fashion. Hum. Brain Mapp. DOI: 10.1002/hbm.23224.

Salari, V., Valian, H., Bassereh, H., Bókkon, I., and Barkhordari, A. (2015). Ultraweak photon emission in the brain. J. Integr. Neurosci. 14, $419-429$.

Salari, V., Scholkmann, F., Bókkon, I., Shahbazi, F., and Tuszynski, J. (2016). The physical mechanism for retinal discrete dark noise: thermal activation or cellular ultraweak photon emission? PLoS ONE 11, e0148336.

Salminen-Vaparanta, N., Vanni, S., Noreika, V., Valiulis, V., Móró, L., Revonsuo, A., Oliverim, M., and Caltagironem, C. (2014). Suppression of extinction with TMS in humans: from healthy controls to subjective characteristics of TMS-induced phosphenes originating in human V1 and V2. Cereb. Cortex 24, 2751-2760.

Scholkmann, F., Kleiser, S., Metz, A.J., Zimmermann, R., Mata Pavia, J., Wolf, U., and Wolf, M. (2014). A review on continuous wave functional near-infrared spectroscopy and imaging instrumentation and methodology. Neuroimage 85, 6-27.

Scott, R.Q., Roschger, P., Devaraj, B., and Inaba, H. (1991). Monitoring a mammalian nuclear membrane phase transition by intrinsic ultraweak light emission. FEBS Lett. 285, 97-98.

Scruggs, J.L., Schmidt, D., and Deutch, A.Y. (2003). The hallucinogen 1-[2,5-dimethoxy-4-iodophenyl]-2-aminopropane (DOI) increases cortical extracellular glutamate levels in rats. Neurosci. Lett. 346, 137-140.

Sczesny-Kaiser, M., Beckhaus, K., Dinse, H.R., Schwenkreis, P., Tegenthoff, M., and Höffken, O. (2016). Repetitive transcranial direct current stimulation induced excitability changes of primary visual cortex and visual learning effects - a pilot study. Front. Behav. Neurosci. 10, 116.

Slawinski, J. (1988). Luminescence research and its relation to ultraweak cell radiation. Experientia 44, 559-571.
Snyder, S.H. and Reivich, M. (1966). Regional localization of LSD in monkey brain. Nature 209, 1093-1095.

Steele, R.H. (2003). Electromagnetic field generation by ATPinduced reverse electron transfer. Arch. Biochem. Biophys. 411, 1-18.

Sun, Y., Wang, C., and Dai, J. (2010). Biophotons as neural communication signals demonstrated by in situ biophoton autography. Photochem. Photobiol. Sci. 9, 315-322.

Takeda, M., Tanno, Y., Kobayashi, M., Usa, M., Ohuchi, N., Satomi, S., and Inaba, H. (1998). A novel method of assessing carcinoma cell proliferation by biophoton emission. Cancer Lett. 127, 155-160.

Tang, R. and Dai, J. (2014a). Biophoton signal transmission and processing in the brain. J. Photochem. Photobiol. B. 139, 71-75.

Tang, R. and Dai, J. (2014b). Spatiotemporal imaging of glutamateinduced biophotonic activities and transmission in neural circuits. PLoS ONE 9, e85643.

Tehovnik, E.J. and Slocum, W.M. (2007). Phosphene induction by microstimulation of macaque V1. Brain Res. Rev. 53, 337-343.

Tehovnik, E.J., Slocum, W.M., Smirnakis, S.M., and Tolias, A.S. (2009). Microstimulation of visual cortex to restore vision. Prog. Brain Res. 175, 347-375.

Tinsley, J.N., Molodtsov, M.I., Prevedel, R., Wartmann, D., Espigulé-Pons, J., Lauwers, M., and Vaziri, A. (2016). Direct detection of a single photon by humans. Nat. Commun. 7, 12172.

Vladimirov, IuA., L'vova, O.F., and Cheremisina, Z.P. (1966). Ultraweak luminescence of mitochondria and its relation to enzymic oxidation of lipids. Biokhimiia 31, 507-515.

Wang, C., Bókkon, I., Dai, J., and Antal, I. (2011). Spontaneous and visible light-induced ultra-weak photon emission from rat eyes. Brain Res. 1369, 1-9.

Watts, B.P., Barnard, M., and Turrens, J.F. (1995). Peroxynitritedependent chemiluminescence of amino acids, proteins, and intact cells. Arch. Biochem. Biophys. 317, 324-330. 